International Journal of Pure and Applied Mathematics

Volume 90 No. 3 2014, 321-333

ISSN: 1311-8080 (printed version); ISSN: 1314-3395 (on-line version)

url: http://www.ijpam.eu

doi: http://dx.doi.org/10.12732/ijpam.v90i3.6

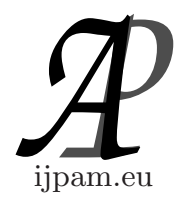

\title{
A COMPARATIVE OF ANALYTICAL AND NUMERICAL SIMULATIONS FOR THE OSCILLATING AIRFLOW \\ IN A HUMAN ORAL AIRWAY
}

\author{
S. Kongnuan ${ }^{1 \S}$, Unchulee Na-Thakuatung ${ }^{2}$, J. Pholuang ${ }^{3}$ \\ 1,2,3 Department of Mathematics and Statistics \\ Faculty of Science and Technology \\ Thammasat University \\ Rangsit Center, Khlong Luang, Pathum Thani, 12121, THAILAND \\ ${ }^{1}$ Centre of Excellence in Mathematics \\ CHE, Si Ayutthaya Rd., Bangkok, 10400, THAILAND
}

\begin{abstract}
Understanding and more accurate simulation of the oscillation airflow are important to aerosolized therapy. To assure the power of simulation by analytical method, in this paper, we present an effective computational method of finite element analysis to compared with the results obtained by an analytical expression in a Fourier series-based form which is presented by S. Kongnuan and J. Pholuang in their previous work. The finite element analysis is implemented by using the commercial software, COMSOL Multiphysics ${ }^{\circledR}$ 3.5a. The airflow fields at different times of each breathing period by both methods are simulated and then compared. The comparative of the results from both methods show a good agreement and agree to the fact of the airflow in a human airway and other related publications.
\end{abstract}

AMS Subject Classification: 65M60, 76D05, 92B05, 97M60

Key Words: human oral airway, Navier-Stokes equations, analytical solution, Fourier series, finite element method, mathematical modelling, oscillating airflow

Received: August 29, 2013

(C) 2014 Academic Publications, Ltd.

$\S$ Correspondence author url: www.acadpubl.eu 


\section{Introduction}

The success of aerosolized therapy depends on the possibility to delivery the proper amount of drug to the appropriate site[1]. It depends on how much we can understand and have more accurate simulation of the oscillation airflow because it defines particle trajectories, transportation and final locations [2], [3], [4], [5] and [6].

The airflow velocity is commonly expressed by the Navier-Stokes equations[3], [7], [8], [9], [10], [11], [12], [13] and [14]. Even though to find a solution of the Navier-Stokes equations by a numerical analysis is quite complex, it is still widely used because there are a lot of high-performance computers nowadays. Many works[15], [16], [17], [18] presented a method of numerical solution for the incompressible Navier-Stokes equations. Furthermore, there are a lot of researches presented a numerical method for such equations and simulate the airflow fields especially in a human respiratory tract[7], [8], [9], [3], [10], [11], [12], [13] and [14].

However, in some specific conditions, the solution of the Navier-Stokes equations can be obtained by an analytical method. To save time and computing resource, some researchers try to find a solution of the Navier-Stokes equations in an analytical expression instead as we can see from many works [19], [20], [21], [22] and [23].

However, analytical expression for the oscillating air flow in a human upper airway has been founded only in[25]. In that work, they did not present any efficient numerical of the same model to compare, they have only compared with other works which are not solved by the same governing equations or the same geometry of the studied domain. In order to assure the effective but save computing resources of the analytical method of a Fourier series-based form which is presented by S. Kongnuan and J. Pholuang in [25] We now propose the method which is already known as an efficient numerically method solving and simulation for the same model and geometry, the finite element analysis to solve and simulation the oscillating air flow in a human airway. The finite element analysis is implemented by using the software, COMSOL Multiphysics ${ }^{\circledR}$ 3.5a. The airflow fields at different times of each breathing period are simulated and then compared. 


\section{Construction of the Model}

\subsection{Parameters and a Human Oral Airway Model}

Due to the complex geometry of a human airway and for convenient to derive an analytical expression of the solution and the reason that we want to show the power of an analytical method than to show on the realistic geometry, we first study in a two-dimensional oral airway model. The model of the domain is started from the beginning of the oral cavity to the end of the trachea in a human upper airway. Their dimensions are summarized as in Table 1[24]. The model has been constructed[25] from the parameters given in Table 1 as depicted in Figure 1(A).

Table 1: Parameters of a human upper airway

\begin{tabular}{|l|c|}
\hline Parameter & Length $(\mathrm{cm})$ \\
\hline The diameter of inlet $\left(L_{1}\right)$ & 3.125 \\
The length of oral cavity $\left(L_{2}\right)$ & 7.0 \\
The diameter of upper trachea $\left(L_{3}\right)$ & 2.5 \\
The length of upper trachea $\left(L_{4}\right)$ & 7.0 \\
The length of lower trachea $\left(L_{5}\right)$ & 9.0 \\
The diameter of outlet $\left(L_{6}\right)$ & 2.0 \\
\hline
\end{tabular}

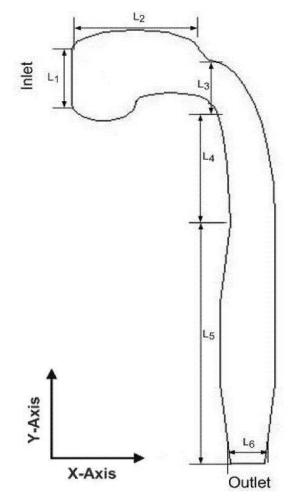

(A)

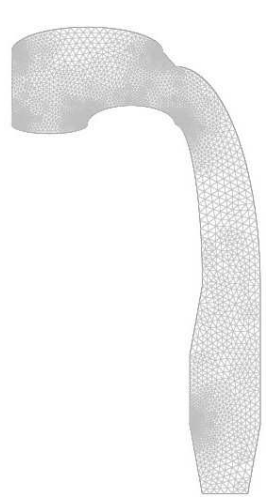

(B)

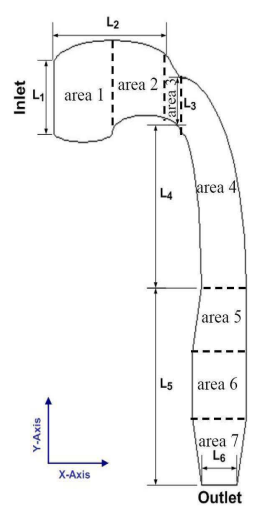

(C)

Figure 1: (A) 2-D human oral airway model; (B) Finite element mesh of the domain; (C) Division of the domain 


\subsection{Governing Equations and Boundary Conditions}

The air is assumed to be an incompressible Newtonian fluid which has constant density and viscosity. For the flow of incompressible Newtonian fluid, most of researches commonly express by the Navier-Stokes equations [3], [7], [8], [9], $[10],[11],[12],[13]$ and [14]. The flow is also assumed that there is no effect from any external force, it is driven only by the oscillating pressure gradient. Here, we are interested in the transportation of the droplets after immediately injected in the oral cavity. At that time, the oral cavity is opened wide, the airflow is assumed to be lamina flow. Therefore, the governing equations for the oscillating two-dimensional airflow are the Navier-Stokes equations and the continuity equation. In the Cartesian coordinates, these equations can be expressed in the following form [25].

$$
\begin{aligned}
\frac{\partial u}{\partial x}+\frac{\partial v}{\partial y} & =0 \\
\rho\left(\frac{\partial u}{\partial t}+u \frac{\partial u}{\partial x}+v \frac{\partial u}{\partial y}\right) & =-\frac{\partial p}{\partial x}+\mu\left(\frac{\partial^{2} u}{\partial x^{2}}+\frac{\partial^{2} u}{\partial y^{2}}\right) \\
\rho\left(\frac{\partial v}{\partial t}+u \frac{\partial v}{\partial x}+v \frac{\partial v}{\partial y}\right) & =-\frac{\partial p}{\partial y}+\mu\left(\frac{\partial^{2} v}{\partial x^{2}}+\frac{\partial^{2} v}{\partial y^{2}}\right)
\end{aligned}
$$

where $u$ and $v$ are the components of the velocity in the Cartesian coordinates and $p$ stands for the pressure. $\rho$ and $\mu$ are the density and dynamic viscosity of the air, respectively.

The non-slip boundary condition, $\boldsymbol{u}=[0,0]$, is assigned to the inner walls. The pressure at the inlet is zero when compared to the outside, while the pressure at outlet is an oscillating function sine of time, we set it be $p(t)=$ $-P \sin (\omega t)$, where $P$ is the amplitude of the oscillating pressure[25]. We now have a boundary value problem which states as follow.

BVP.: Find $u, v$ and $p$ such that all equations(1)-(3) are satisfied and all boundary conditions are satisfied on the relevant boundary segments.

\section{Method of Numerical Solution}

The boundary valued problem of the oscillating airflow as stated in the previous section is a transient and nonlinear problem. To solve this problem by a numerical method, we use the finite element based software, COMSOL Multiphysics ${ }^{\circledR}$ 
3.5a. A 2-D human oral airway model as depicted in Figure 1(A) is firstly constructed in Draw mode. Then the incompressible Navier-Stokes equations is imposed to the model in Multiphysics mode. In Mesh mode, the 2-D human oral airway domain is discretized into a finite number of small elements of 7484 triangles which connected by 3910 node points of lagrange order 2 as shown in Figure 1(B). Since for the air has very low density, we need to set a value of Isotropic diffusion in Artificial Diffusion for Navier-Stokes equations in Subdomain settings of Physics mode. This value must be adjusted until it is suitable for the mesh size without this setting, solving would not have succeeded. The boundary conditions as previous stated is also set for Boundary settings in Physics mode. In Solve mode, the tolerance of convergence is set to be $10 \times 10^{-7}$ and the Direct(UMFPACK) is assigned to the linear system solver in Solver Parameters. The obtained results for the oscillating airflow are presented in the section of Results and discussion.

\section{Method of Analytical Solution}

In this part, we use the analytical method of a Fourier series-based form which is presented by S. Kongnuan and J. Pholuang in [25]. To derive an analytical expression of the airflow velocity, the 2-D oral airway domain is divided into subregions as shown in Figure 1(C). Firstly, the original shape of the subregions is transformed into a rectangular channel as an example shown in Figure 2.

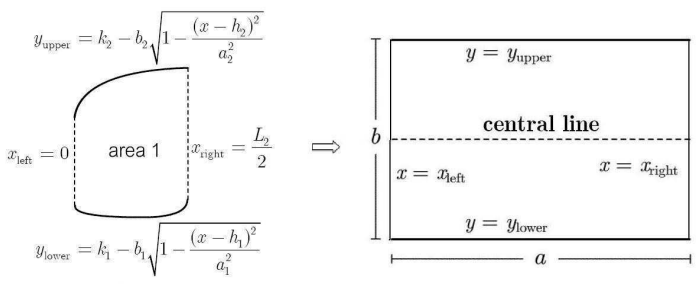

Figure 2: Transformation of a region

The airflow is then transformed to be the flow in straight channels. We only brief the finding an analytical solution of the airflow in the horizontal areas. For equations (1)-(3), there are three partial differential equations with three unknowns $u, v$ and $p$ as functions of three independent variables $x, y$ and $t$. Assuming fully developed flow on these regions, $v=0$ and $u=u(t, y)$, the continuity equation is satisfied and when developed flow conditions across the 
$x$-axis; therefore, equation (3) is omitted. From the boundary conditions, at the leftmost of the domain, $x=0, p(0, y, t)=0$; and at the rightmost of the domain, $x=a, p(a, y, t)=p(t)$. Then the following partial differential equations satisfied:

$$
\frac{\partial u}{\partial t}=-\frac{1}{\rho} \frac{\partial p}{\partial x}+\nu\left(\frac{\partial^{2} u}{\partial y^{2}}\right), \quad-\frac{\partial p}{\partial x}=\frac{P}{a} \sin (\omega t),
$$

where $\frac{P}{a}$ is the amplitude of the imposed pressure gradient, $a$ is the length on $x$-axis of the considered region, $\nu=\frac{\mu}{\rho}$ is its kinematic viscous coefficient and $\omega$ is the cyclic frequency of the oscillating pressure gradient. The solution $u$ is assumed to be periodic so that:

$$
u(y, t)=u_{s}(y) \sin (\omega t)+u_{c}(y) \cos (\omega t) .
$$

Dimensionless variables $x, y, u$ and $\alpha$ are introduced as

$$
\tilde{x}=\frac{x}{a}, \quad \tilde{y}=\frac{y}{b}, \quad \tilde{u}=\frac{u}{P b^{2}} \mu a, \quad \alpha=b \sqrt{\frac{\omega \rho}{\mu}},
$$

where $b$ is the length on $y$-axis of the considered region. Then each region is transformed to be a one-unit rectangular region.

Equation (4) together with equation (5) are reduced to a system of nonhomogeneous Helmholtz equations in one dimension[26]:

$$
\alpha^{2} \tilde{u}_{s}=\frac{d^{2} \tilde{u}_{c}}{d \tilde{y}^{2}}, \quad-\alpha^{2} \tilde{u}_{c}=1+\frac{d^{2} \tilde{u_{s}}}{d \tilde{y}^{2}},
$$

where $\alpha$ is the reduced frequency. The boundary conditions for $\tilde{u}_{s}$ and $\tilde{u}_{c}$ result from using the boundary conditions and oscillating flow assumption (5):

$$
\tilde{u}_{s}(0)=0, \quad \tilde{u}_{c}(0)=0, \quad \tilde{u}_{s}(1)=0, \quad \tilde{u}_{c}(1)=0 .
$$

For equations (7), the analytical solution which satisfies the boundary conditions (8), can be determined by using a Fourier series of $\tilde{u}_{s}, \tilde{u}_{c}$ for $\tilde{y}$. Hence, $\tilde{u}_{s}, \tilde{u}_{c}$ and 1 are expressed[27] as:

$$
\begin{aligned}
\tilde{u}_{s} & =\sum_{k=1}^{\infty} A_{k} \sin (k \pi \tilde{y}), \quad \tilde{u}_{c}=\sum_{k=1}^{\infty} B_{k} \sin (k \pi \tilde{y}), \\
1 & =\sum_{k=1}^{\infty} C_{k} \sin (k \pi \tilde{y}),
\end{aligned}
$$


where $C_{k}$ can be calculated by

$$
C_{k}=\frac{\int_{0}^{1} \sin (k \pi \tilde{y}) d \tilde{y}}{\int_{0}^{1} \sin ^{2}(k \pi \tilde{y}) d \tilde{y}}=\frac{2}{k \pi}\left(1-(-1)^{k}\right) ; \quad k=1,2,3, \cdots .
$$

Substituting the series (9)-(10) in the system of differential equations (7), the unknown coefficients $A_{k}$ and $B_{k}$ are as follows:

$$
A_{k}=C_{k}\left(\frac{\pi^{2} k^{2}}{\alpha^{4}+k^{4} \pi^{4}}\right), B_{k}=C_{k}\left(\frac{-\alpha^{2}}{\alpha^{4}+k^{4} \pi^{4}}\right) ; k=1,2,3, \cdots .
$$

The resulting periodic velocity can be written as:

$$
\tilde{u}=\tilde{u}_{a} \cos (\omega t), \quad \tilde{u}_{a}=\sqrt{\tilde{u}_{s}^{2}+\tilde{u}_{c}^{2}},
$$

where $\tilde{u}_{a}$ is the amplitude resulting from the expression of $\tilde{u}$. We can obtain the airflow velocity $u$ by substituting $\tilde{u}$ back into (6), we yield $u=\frac{P b^{2}}{\mu a} \tilde{u}$. The airflow velocity for the vertical regions is in the same fashion.

\section{Results and Discussion}

Based on medical literatures, the breathing period is about $4 s[12]$, therefore, we use the cyclic frequency $\omega=\frac{\pi}{2}$. The analysis is carried out with $\rho=1.148$ $\mathrm{kg} / \mathrm{m}^{3}, \mu=1.82 \times 10^{-5} \mathrm{~Pa} \cdot \mathrm{s}$ and the amplitude of the oscillating pressure $P=133.32 P a[25]$.

The arrow plots of the velocity fields at $t=1.5 \mathrm{~s}, t=2.05 \mathrm{~s}$ and $t=3.5 \mathrm{~s}$ of each breathing period are shown in Figures 3(A)-3(C) for the numerical method and Figures 4(A)-4(C) for the analytical method. They show a good agreement that when $t=1.5 \mathrm{~s}$ the air flows into the oral airway which corresponds to the pulmonary relaxation. In contrast, when $t=2.05 \mathrm{~s}$ and $t=3.5 \mathrm{~s}$, the air flows out the oral airway which corresponds to pulmonary contraction. The high speed velocities from both methods occur in the central area and reduce to the zero for the area which is more close to the walls.

The contour plots of the velocity field at $t=1.5 \mathrm{~s}, t=2.05 \mathrm{~s}$ and $t=3.5 \mathrm{~s}$ of each breathing period are shown in Figures 5(A)-5(C) for the numerical method and Figures $6(\mathrm{~A})-6(\mathrm{C})$ for the analytical method. It is the same, their results also show agreements that the velocity profiles become flat in the central area. This shows a boundary layer behavior with a high velocity gradient close to the boundaries. Their amplitudes of velocities always show the maximum value in the central area and reduce to the zero value for the area which is more close 

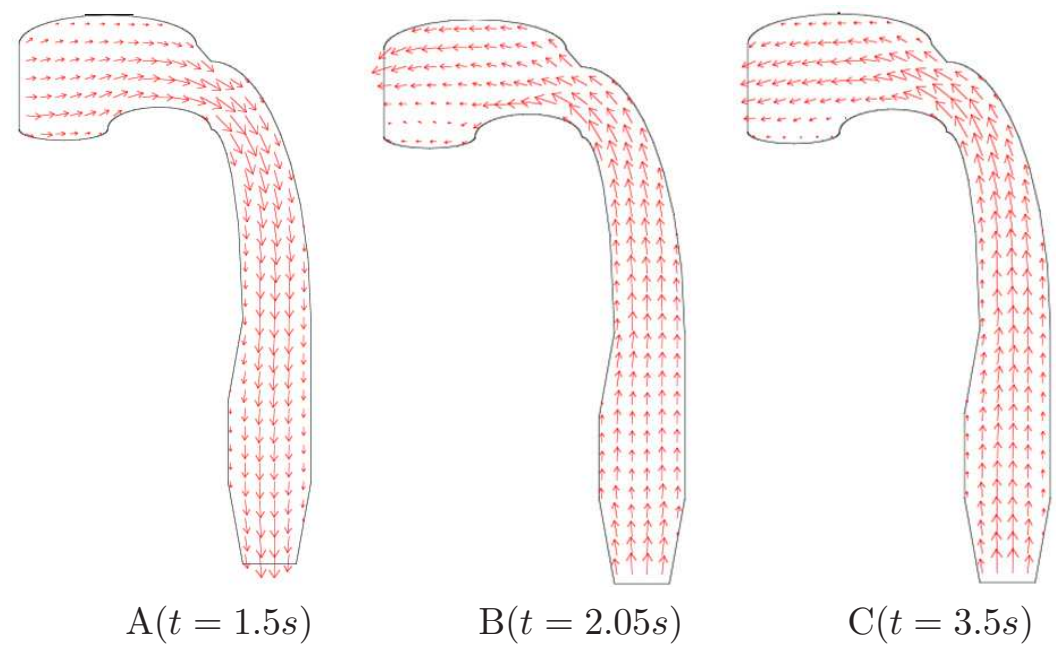

Figure 3: Arrow plots of the velocity field $(\mathrm{cm} / \mathrm{s})$ by the numerical method
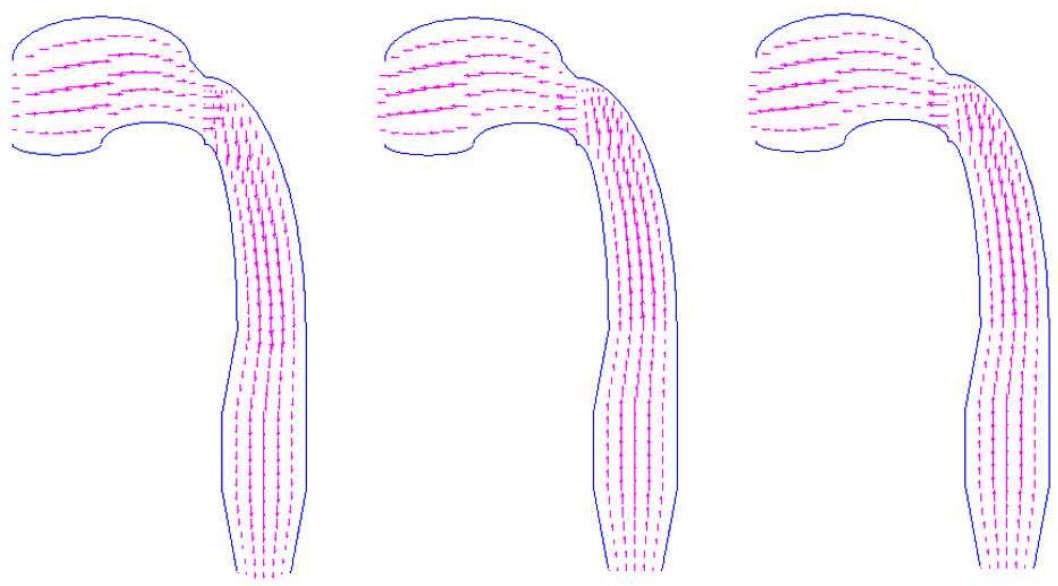

$$
\mathrm{A}(t=1.5 s)
$$

$$
\mathrm{B}(t=2.05 s)
$$$$
\mathrm{C}(t=3.5 s)
$$

Figure 4: Arrow plots of the velocity field $(\mathrm{cm} / \mathrm{s})$ by the analytical method

to the walls which correspond to the arrow plots. The velocities at different times are different and when $t=2.05 \mathrm{~s}$ the amplitudes of the velocity are close 

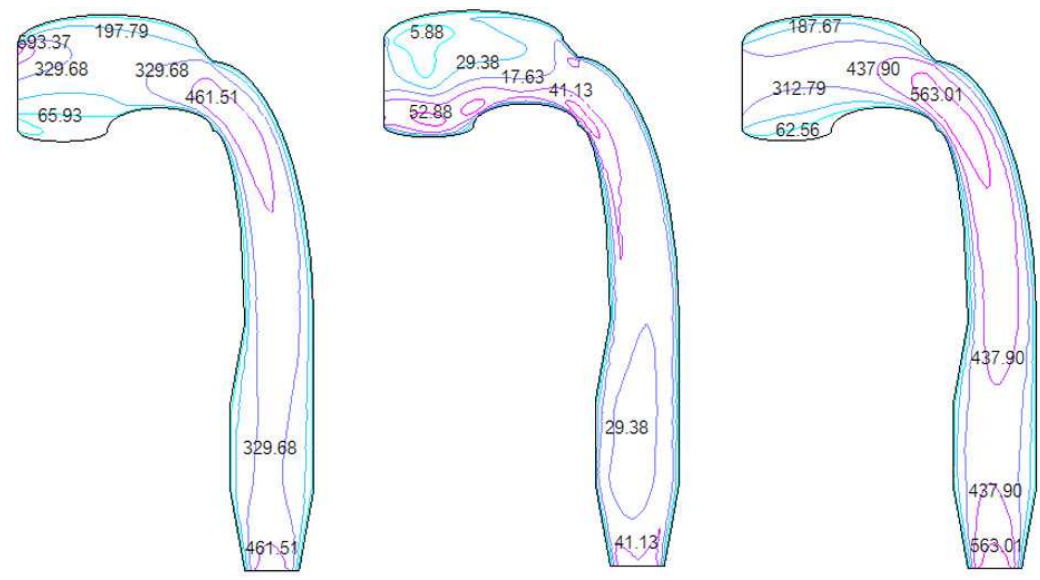

$$
\mathrm{A}(t=1.5 s)
$$

$\mathrm{B}(t=2.05 s)$

$$
\mathrm{C}(t=3.5 s)
$$

Figure 5: Contour plots of the airflow field $(\mathrm{cm} / \mathrm{s})$ by the numerical method
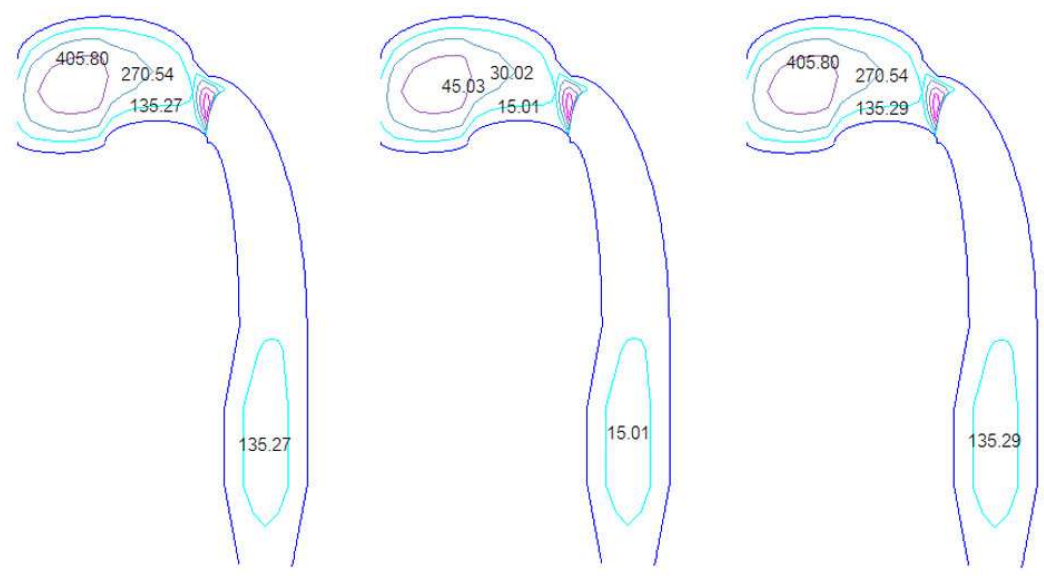

$$
\mathrm{A}(t=1.5 s)
$$

$\mathrm{B}(t=2.05 s)$

$$
\mathrm{C}(t=3.5 s)
$$

Figure 6: Contour plots of the airflow field $(\mathrm{cm} / \mathrm{s})$ by the analytical method

to zero. Although, the obtained values of velocities are a little bit different by each method but they are in the same range of $[0,900] \mathrm{cm} / \mathrm{s}$ which agree to other publications [4] and [24]. 


\section{Conclusions}

Two effective methods, the method of finite element analysis implemented by using COMSOL Multiphysics ${ }^{\circledR}$ 3.5a; and the method of analytical expression which is in a Fourier series-based form are carried out on a two-dimensional geometry of a human oral airway model. The obtained airflow profiles from both methods are reasonable and feasible. When the comparative has made, they show a good agreement and they also agree to the fact that the laminar flow has the maximum velocity in the central area and reduces to the zero value close to the walls [28] and agree to other related publications [4], [12] and [24] - However, when computational resources and complexity of implementation are considered, the analytical method has more advantages than the numerical method. Therefore, the presented analytical expression is satisfied as we expected.

\section{Acknowledgments}

This research is supported by the Centre of Excellence in Mathematics, the Commission on Higher Education, Thailand. The authors are also very grateful to Department of Mathematics and Statistics, Thammasat University for giving the facilities support and Department of Mathematics, Faculty of Science, Mahidol University for the commercial software, COMSOL Multiphysics ${ }^{\circledR}$ 3.5a support.

\section{References}

[1] L. Gradon and J.C. Marijnissen, Optimization of Aerosol Drug Delivery, Kluwer Academic Publishers, Netherlands(2003).

[2] M. Giroux, P. Hwang, A.K. Prasad, Controlled particle dispersion, applying verticle flow to optimize nasal drug deposition, Drug delivery Technology, 5(2005), 44-49.

[3] H. Tang, J.Y. Tu, H.F. Li, B. Au-Hijleh, C.C. Xue, C.G. Li, Dynamic analysis of airflow features in a 3D real-anatomical geometry of the human nasal cavity, 15th Australasian Fluid Mechanics Conference, Australia, 1317 December, 2004. 
[4] Z. Zhang and C. Kleinstreuer, Airflow structures and nano-particle deposition in a human upper airway model, Journal of Computational Physics, 198(2004), 178-210.

[5] Q. Xu, F.Y. Leong and C.H. Wang, Transport and deposition of inertial aerosols in bifurcated tubes under oscillatory flow, Chemical Engineering Science, 64(2008), 830-846.

[6] L. Zhang, H. Cheng, C. Zhang, Z. Xu, J. Ye, Deposition fraction of aerosol particles in a human oral airway model on stable condition, Aerosal and Air Quality Research, 6(2006), 259-267.

[7] G.M. Allen, B.P. Shortall, T. Gemci, T.E. Corcoran, and N.A. Chigier, Computational simulations of airflow in an in vitro model of the pediatric upper airways, Journal of Biomechanical Engineering, 126(5)(2004), 604613.

[8] Y. Lee, Finite Element simulation of the oscillatory flow in a channel with the heat transfer through a hot bump, Proceeding of the COMSOL Multiphysics User's Conference, Boston, 2005.

[9] X.Y. Luo, J.S. Hinton, T.T. Liew and K.K. Tan, LES modeling of flow in a simple airway model, Medical engineering $\&$ Physics, 26(2004), 403-413.

[10] K. Wang, T.S. Denney, E.E. Morrison, V.J. Vodyanoy, Numerical simulation of air flow in the human nasal cavity, Proceedings of the 2005 IEEE Engineering in Medicine and Biology 27th Annual Conference Shanghai, China, 1-4 September, 2005 , 5607-5610.

[11] J. Wen, K. Inthavong, Z.F. Tian, J.Y. Tu, C.L. Xue and C.G. Li, Airflow patterns in both sides of a realistic human nasal cavity for laminar and turbulent conditions, 16th Australasian Fluid Mechanics Conference Crown Plaza, Gold Coat, Australia, 2-7 December, 2007, 68-73.

[12] L. Zhang, H. Cheng, C. Zhang, Z. Xu and J. Ye, Deposition fraction of aerosol particles in a human oral airway model on stable condition, Aerosol and Air Quality Research, 6(2006), 259-267.

[13] Z. Zhang, C. Kleinstreuer, C.S. Kim, Effects of curved inlet tubes on air flow and particle deposition in bifurcating lung models, Journal of Biomechanics, 34(2001), 659-669. 
[14] Z. Zhang and C. Kleinstreuer, Airflow structures and nano-particle deposition in a human upper airway model, Journal of Computational Physic, 198(2004), 178-210.

[15] A.J. Chorin, Numerical solution of the Navier-Stokes equations, $A E C$ Research and Development Report, New York Univ., 1968.

[16] F.C. Thames, J.F. Thompson, and C. Wayne Mastin, Numerical solution of the Navier-Stokes equations for arbitrary two-dimensional airfoils, Mississippi state University,1983.

[17] M. Quadrio and P. Luchini, The numerical solution of the incompressible Navier-Stokes equations on a low-cost,Dedicated parallel computer, 2004.

[18] S.A. Salem, On the numerical solution of the incompressible Navier-Stokes equations in primitive variables using grid generation techniques, Matematical and Computational Applications, 11(2)(2006), 127-136.

[19] H.I. Andersson, An exact solution of the Navier-Stokes equations for magnetohydrodynamic flow, Acta Mechanica, 113(1995), 241-244.

[20] S. Tsangaris, N.W. Vlachakis, Exact solution of the Navier-Stokes equations for the oscillating flow in duct of a cross-section of right-angled isosceles triangle, Z. angew. Math. Phys., 54(2003), 1094-1100.

[21] S. Otarod and D. Otarod, Analytical solution for Navier-Stokes equations in two dimensions for laminar incompressible flow, Fluid Dynamics; Classial Physics, 2006.

[22] M.R. Mohyuddin, A.M. Siddiqui, T. Hayat, J. Siddqui, S. Asghar, Exact solutions of time-dependent Navier-Stokes equations by HodographLegendre transformation method, Tamsui Oxford Journal of Mathematical Sciences, 24(3)(2008), 257-268.

[23] M. Emin, C. Erdem, An Analytical Solution of the Navier-Stokes Equation for Flow over a Moving Plate Bounded by Two Side Walls, Journal of Mechanical Engineering, 55(2009)12, 749-754.

[24] C.G. Ball, M. Uddin, A. Pollard, Mean flow structures inside the human upper airway, Flow Turbulence Combust, 81(2008), 155-188.

[25] S. Kongnuan and J. Pholuang, A Fourier Series-Based Analytical Solution for the oscillating Airflow in a Human Respiratory Tract, International journal of pure and applied mathematic, 78(5)(2012), 721-733. 
[26] H. Rosu and J.L. Romero, Ermakov approach for the one-dimensional Helmholtz Hamiltonian, Nuovo Cimento, 114(1999), 569-574.

[27] N. Oliver, Wave Equations, Fourier Series and Wave Models[Online], http://cecs.anu.edu.au/seminars/media/ 819_05_03_09_Oliver_Nagy_slides.pdf, Retrieved November 11, 2010.

[28] B.R. Munson, D.F. Young, T.H. Okiishi, Fundamental of fluid mechanics, John Wiley \& Sons, Inc., USA (1990). 
